Sir,

\section{Ocular leishmaniasis}

Leishmaniasis is a parasitic infection caused by the protozoon Leishmania and transmitted by female Phlebotomus sand-flies. Hosts include cats, dogs, rodents and humans. The disease is endemic to Middle and South America, Africa, the Middle East, India and many Mediterranean countries. ${ }^{1}$ Here the clinical and histopathological features of a case of ocular leishmaniasis involving the lids and conjunctiva are discussed.

\section{Case report}

A 54-year-old woman presented to our clinic with the complaints of secretion and pain in the left eye and a lesion of the lower left eyelid. The patient had received variable topical antibiotic regimens previously but the lesion had not improved. Ocular examination revealed an ulcerative lesion located on the lateral part of the lower left eyelid, reaching to the fornix. The lesion was 8 $3 \mathrm{~mm}$ in size, with oedematous edges. The tarsal conjunctiva was hyperaemic and oedematous in the region of the lesion (Fig. 1). Biomicroscopic examination revealed mucopurulent secretion, punctate epithelial erosions of the cornea and early cortical cataracts. The fundus examinations and intraocular pressures were normal bilaterally. Visual acuity was 1.0 in the right eye and 0.9 in the left eye.

There was no other nodular lesion or preauricular lymphadenopathy in the face. Systemic examination excluded lymphadenopathy, hepatomegaly or splenomegaly. Laboratory investigations revealed high levels of total cholesterol and an increased number of active $T$ cells. There was no other pathological finding.

Excisional biopsy of the lesion was performed as the lesion was similar to squamous cell carcinoma clinically. Histopathological examination of the haematoxylineosin stained biopsy material revealed inflammatory cells consisting mainly of plasma cells, and also of histiocytes and lymphocytes among the proliferated vascular structures under the epithelium. Many round, dot-like microorganisms, mostly extracellular and less common in the histiocytes, were present in almost all microscopic fields (Fig. 2). This histological appearance was consistent with leishmaniasis. To confirm the diagnosis and exclude the possibility of Histoplasma, which is an intracellular microorganism, the biopsy material was also stained with Giemsa dye and Gomori's methamine silver stain (MSS). The microorganisms stained with Giemsa but not with MSS. With these findings, the patient was diagnosed as having ocular leishmaniasis. Allopurinol was given 200 $\mathrm{mg} / \mathrm{kg}$ in four divided doses orally for 15 days. After the corneal lesions had healed with topical antibiotics, topical steroids were used. Liver and kidney function tests were performed before the therapy and at 1 week, 3 weeks, 3 months and 6 months after the treatment.

At the patient's last visit, 10 months later, the lesion in the lower lid was healed with scarring. There was no lid deformity.

\section{Comment}

Leishmaniasis is accepted as one of the six important tropical diseases by the World Health Organization. Every year, about 2 million new cases are recognised and 400 million people are at risk. ${ }^{2}$ Cutaneous leishmaniasis was endemic to the south-eastern part of Turkey in the 1950s but its incidence has been decreased by effective vector control. Since the 1980 s the incidence has increased again, and in 1995 there were 2809 reported cases of cutaneous leishmaniasis.

Leishmaniasis is classified into Old and New World cutaneous leishmaniasis, mucocutaneous and visceral leishmaniasis. Cutaneous leishmaniasis is rarely fatal and is characterised by single or multiple ulcers, usually on the face or extremities. ${ }^{1,3,4}$ The initial lesion is a small, itchy papule. This later consists of solitary, crusty, ulcerated granulomas. These lesions usually heal spontaneously with a scar within a few months. L. major, L. tropica, $L$. infantum and L. aethiopica might be isolated from the lesions. Eyelids are affected in $2-5 \%$ of cases. ${ }^{1}$

Ocular leishmaniasis produces ulcerative or granulomatous lesions on the lids and conjunctiva, blepharoconjunctivitis and interstitial keratitis. ${ }^{5-7}$ Conjunctival invasion of leishmaniasis is by contagious, haematogeneous or direct inoculation. ${ }^{5}$ Long-term complications of ocular leishmaniasis are uveitis, ${ }^{6}$ dacryocystitis, interstitial keratitis, ${ }^{8}$ corneal ulceration ${ }^{6}$ and lid deformities. ${ }^{3,7}$ It is assumed that ocular leishmaniasis could be more common than reported in the literature as leishmanial lesions are often confused

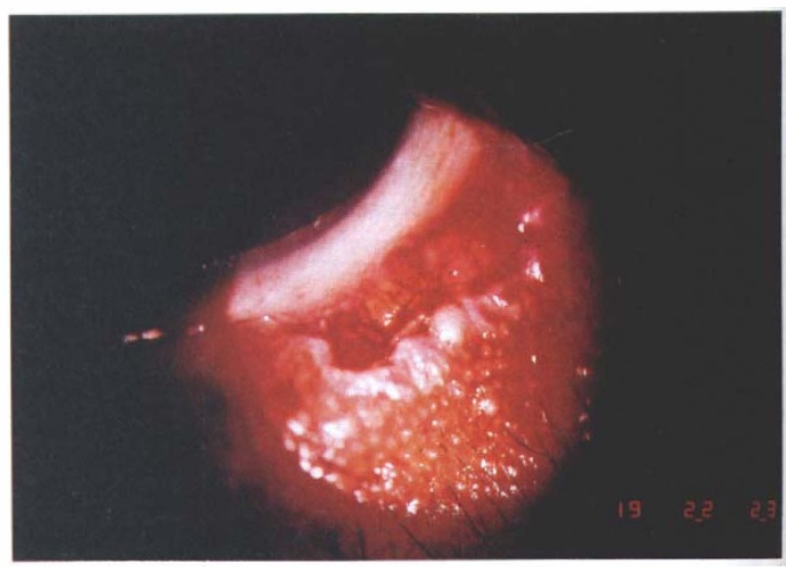

Fig. 1. Ocular leishmanial lesion involving the lower left eyelid. 


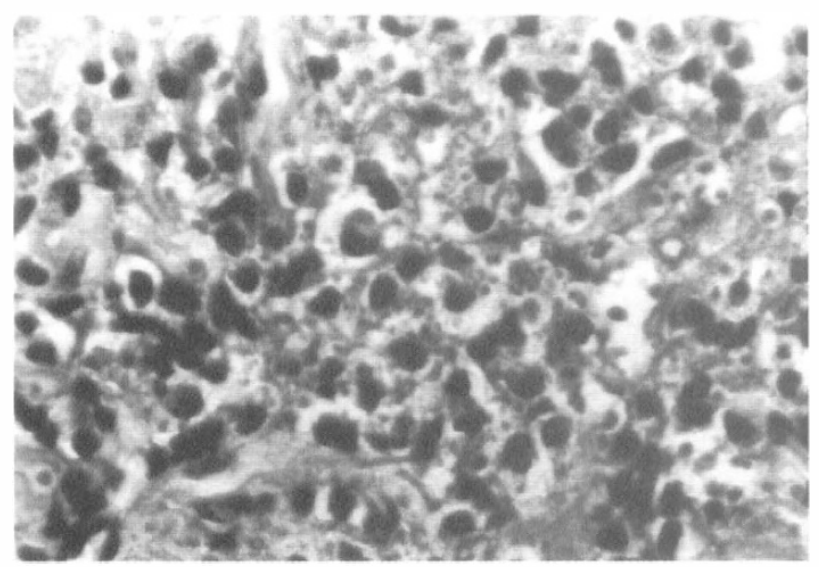

Fig. 2. Histopathological appearance of the lesion.

with chalazion, epidermoid cyst, dacryocystitis and tumours. ${ }^{1,6}$ Ocular leishmaniasis of the lower lid may heal spontaneously.

The treatment of ocular leishmaniasis includes prevention of secondary infections, intralesional or systemic antimony compounds (meglumine antimoniate) and allopurinol. ${ }^{1,3-9}$ Systemic interferon has been shown to be effective in the treatment of cutaneous leishmaniasis. ${ }^{10}$ We used allopurinol in the treatment of our case since antimony compounds can lead to serious side-effects such as fatal cardiac arrythmias, hepatoxicity and nephropathy, in addition to gastrointestinal upset such as nausea and vomiting. Additionally, it is difficult to supply antimony compounds in Turkey.

In conclusion, ocular leishmaniasis should be considered in the differential diagnosis of ulcerations, abscesses and nodules of the eyelids and ulcerated lesions of the conjunctiva, especially in endemic areas. Cicatrising sequelae of ocular leishmaniasis can be prevented by early diagnosis and treatment.

\section{References}

1. O'Neill DP, Deutsch J, Carmichael AJ, Taylor R. Eyelid leishmaniasis in a patient with neurogenic ptosis. $\mathrm{Br} \mathrm{J}$ Ophthalmol 1991;75:506-7.

2. Control of leishmaniasis. Report of a WHO Expert Committee. World Health Organization technical report series 793. Geneva: WHO, 1990.

3. Satici A, Gürler B, Gürel MS, Aslan G, Oguz H. Mechanical ptosis and lagophthalmos in cutaneous leishmaniasis. $\mathrm{Br} \mathrm{J}$ Ophthalmol 1998;82:975.

4. Chu FC, Rodrigues MM, Cogan DG, Neva AF. Leishmaniasis affecting the eyelids. Arch Ophthalmol 1983;101:84-91.

5. Nandy A, Addy M, Chowdhury AB. Leishmanial blepharoconjunctivitis. Trop Geogr Med 1991;43:303-6.

6. Morgan G. Case of cutaneous leishmaniasis of the lid. Br J Ophthalmol 1965;49:542-5.

7. Chaudhry IA, Hylton C, DesMarchais B. Bilateral ptosis and lower eyelid ectropion secondary to cutaneous leishmaniasis. Arch Ophthalmol 1998;116:1244-5.

8. Martinez S, Marr JJ. Allopurinol in the treatment of American cutaneous leishmaniasis. N Engl J Med 1992;326:741-4.

9. Mahmoud AAF. The challenge of intracellular pathogens. $\mathrm{N}$ Engl J Med 1992;326:761-2.

10. Kolde G, Luger T, Sorg C, Sunderkotter C. Successful treatment of cutaneous leishmaniasis using systemic interferon-gamma. Dermatology 1996;192:56-60.
Yildiz Özdemir ${ }^{1}$

Sezer Kulaçoḡlu²

C. Banu Coşar ${ }^{1}$

Feyza Onder ${ }^{1}$

Gülcan Kural

${ }^{1}$ Eye Clinic 1

Ankara Numune Hospital

Ankara, Turkey

${ }^{2}$ Department of Pathology

Ankara Numune Hospital

Ankara, Turkey

Yildiz Özdemir, MD

Sancak mah.233.Sok.6/12

Cankaya Yildiz 06550

Ankara, Turkey

Tel: +903124388460

Fax: +903862141615

Sir,

Endoscopic transnasal removal of orbital foreign body Facial trauma involving breakable objects may result in concealed orbital foreign bodies. These may be missed unless specifically sought, and excluded with careful clinical examination and imaging techniques. We report an unusual case of an orbital foreign body with no apparent entry wound. The object was subsequently discovered to have entered the orbit via a nostril. To our knowledge, endoscopic transethmoidal removal of a posterior orbital foreign body via the nares has not previously been reported.

\section{Case report}

A 40-year-old man was admitted following an assault with a blunt object. He had a full-thickness left upper lip laceration and a right-sided $4 \mathrm{~mm}$ proptosis with associated periorbital bruising. On examination, unaided visual acuity was $6 / 9$ in the affected right eye, $6 / 6$ in the left. There was limitation in right abduction and depression. No trauma to the anterior segment or relative afferent pupillary defect was detected. Colour vision and fundoscopy was normal. There was no facial anaesthesia or surgical emphysema, nor any evidence of cerebrospinal fluid rhinorrhoea. Facial radiographs revealed a large, deeply placed radio-opaque foreign body within the right orbit (Fig. 1). Examination under general anaesthesia was undertaken and the lip wound repaired. Periorbital and lid examination did not demonstrate any breach in the skin or conjunctiva as a possible route of entry.

To accurately locate the foreign body and plan orbital surgery a CT scan was performed. This revealed a $41 \mathrm{~mm}$ object lying superior to the right lateral rectus and optic nerve, with the medial end of the object transfixing the medial rectus and lying partly within the ethmoidal sinus. The scans showed no evidence of orbital roof fracture. Examination by an otolaryngologist confirmed mid-nasal septal lacerations, trauma to the middle 